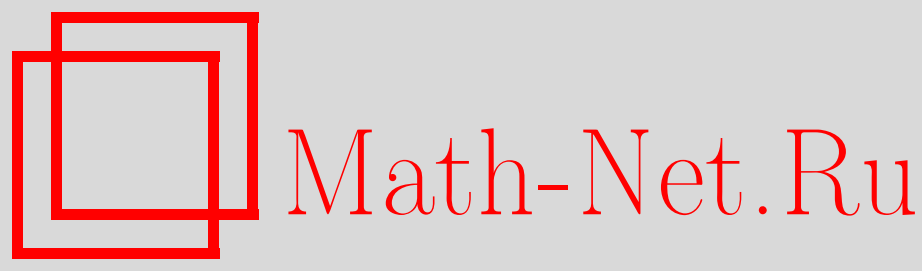

С. В. Гуц, Квазиклассический анализ рождения пар в пространстве де Ситтера, ТМФ, 2014, том 180, номер 1, 72-85

DOI: https://doi.org/10.4213/tmf8629

Использование Общероссийского математического портала Math-Net.Ru подразумевает, что вы прочитали и согласны с пользовательским соглашением http://www.mathnet.ru/rus/agreement

Параметры загрузки:

IP : 3.85 .183 .62

26 апреля 2023 г., 15:10:08

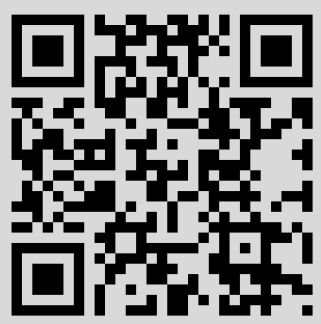




\title{
КВАЗИКЛАССИЧЕСКИЙ АНАЛИЗ РОЖДЕНИЯ ПАР В ПРОСТРАНСТВЕ ДЕ СИТТЕРА
}

\begin{abstract}
Рассмотрена теория скалярного массивного поля в пространстве де Ситтера. С использованием подхода инстантонов на мировых линиях вычислена вероятность рождения пар в пределе слабых полей. В дополнение к экспоненциальному получен преэкспоненциальный фактор. В рамках этого метода вероятность рождения пар для нечетномерных пространств обращается в ноль. Найден основной вклад в двухточечный коррелятор в $\phi^{3}$-теории.
\end{abstract}

Ключевые слова: квантовые поля в кривом пространстве, пространство де Ситтера.

DOI: $10.4213 / \operatorname{tmf} 8629$

\section{1. ВВЕДЕНИЕ}

Квазиклассические методы представляют собой мощный инструмент исследования непертурбативных явлений в современной квантовой теории поля. Распад ложного вакуума является одним из наиболее хорошо изученных примеров [1]-[4]. Решая уравнения движения, мы находим решение, интерполирующее между истинным и ложным вакуумом. Вероятность распада пропорциональна евклидову действию данного решения.

Схожие методы применяются для исследования рождения частиц во внешнем поле. Наиболее хорошо изучен эффект Швингера - спонтанное рождение электронпозитронной пары в электрическом поле [5]-[7]. Вероятность рождения пары может быть выражена в терминах однопетлевого эффективного действия. Решения седлового уравнения, именуемые инстантонами на мировых линиях, можно интерпретировать как траектории частиц, движущихся в евклидовом пространстве.

Сильное экспоненциальное подавление таких процессов стало причиной исследования индуцированных процессов. Для ряда случаев такие процессы оказались более вероятными, чем их спонтанные аналоги [8]-[10].

Мы рассматриваем аналогичные процессы рождения пар во внешнем гравитационном поле. В последнее время эта проблема исследовалась как при помощи квазиклассических методов [11], так и при помощи кинетического уравнения [12], [13].

* Институт теоретической и экспериментальной физики, Москва, Россия. E-mail: guts@itep.ru 
В настоящей работе исследуется модельный пример самодействующей скалярной теории поля в пространстве де Ситтера. Используя евклидово действие и инстантоны на мировых линиях, мы анализируем ширину спонтанного распада для невзаимодействующей теории. Далее мы покажем, что этот метод можно модифицировать для получения мнимой части двухточечного коррелятора, который описывает индуцированное рождение частиц.

Статья разбита на разделы следующим образом. В разделе 2 мы рассматриваем спонтанное рождение частиц для двумерного пространства де Ситтера. Мы объясняем, как поступать с новым типом расходимостей, и выводим формулу для скорости рождения частиц в пределе слабого поля. В разделе 3 мы вычисляем основной вклад в двухточечный коррелятор в двумерном пространстве де Ситтера. Наконец, в разделе 4 мы выполняем эти вычисления для произвольной размерности $d$ пространства и в разделе 5 подводим итоги.

\section{2. СПОНТАННОЕ РОЖДЕНИЕ ПАР}

В квантовой теории поля евклидово однопетлевое эффективное действие для действительной массивной частицы $\phi$ с потенциалом $U(f)$ может быть записано как

$$
\Gamma[\phi]=-\frac{1}{2} \operatorname{Tr} \ln \left(-\square+m^{2}+U^{\prime \prime}(\phi)\right) .
$$

Используя формулу

$$
-\operatorname{Tr} \ln A=\int_{0}^{\infty} \frac{d T}{T} \operatorname{Tr} e^{A T},
$$

мы удаляем не зависящие от $\phi(x)$ слагаемые и вычисляем функциональный след в $x$-пространстве. Это дает нам

$$
\Gamma[\phi]=\int_{0}^{\infty} \frac{d T}{T} \int d^{D} x\left\langle x\left|e^{-T\left(-\square+m^{2}+U^{\prime \prime}(\phi)\right)}\right| x\right\rangle .
$$

Правую часть этого уравнения можно выразить в терминах интеграла по путям так же, как это делается для уравнения Шредингера (более подробно это описано в работе [14]):

$$
\Gamma[\phi]=\int_{0}^{\infty} \frac{d T}{T} e^{-m^{2} T} \int_{x(1)=x(0)} \mathcal{D} x \exp \left[-\int_{0}^{1} d \tau\left(\frac{\dot{x}^{2}}{4 T}+T U^{\prime \prime}(\phi(x))\right)\right] .
$$

Здесь мы интегрируем по всем замкнутым путям $x^{\mu}(\tau)$ в евклидовом пространстве, периодическим по $\tau$ с периодом 1. Эффективное действие $\Gamma_{\text {Eucl }}[\phi]$ - это функционал, зависящий от классического поля $\phi(x)$.

Хорошо изучен схожий случай со скалярной заряженной частицей в классическом электромагнитном потенциале $A_{\mu}(x)$, отвечающем электрическому полю в пространстве Минковского, когда однопетлевое эффективное действие имеет непертурбативную мнимую часть, отвечающую рождению частиц. Физическая интерпретация этого факта состоит в том, что амплитуда вакуумной устойчивости связана с эффективным действием для пространства Минковского, $\langle 0 \mid 0\rangle=e^{i \Gamma_{\text {Mink }}}$, поэтому

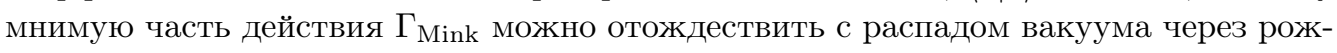
дение частиц, так что вероятность рождения частиц может быть записана в виде

$$
P_{\text {prod }}=1-e^{-2 \operatorname{Im} \Gamma_{\text {Mink }}} \approx 2 \operatorname{Im} \Gamma_{\text {Mink }} .
$$


В слабом постоянном электрическом поле $E$ мнимую часть действия можно представить как

$$
\operatorname{Im} \Gamma_{\text {Mink }} \sim V_{4}^{\operatorname{Mink}} \frac{e^{2} E^{2}}{16 \pi^{3}} \exp \left[-\frac{m^{2} \pi}{e E}\right] .
$$

В работе [6] было показано, как, используя квазиклассическое приближение, вычислить этот лидирующий вклад в мнимую часть.

Схожий процесс происходит в неплоской метрике [11], [12]. Такие процессы чаще исследуют в терминах in-out вакуума [15], чем в терминах инстантонов на мировых линиях [16]. Это побуждает нас применить метод инстантонов на мировых линиях для исследования неустойчивости пространства де Ситтера. Ключевое различие поворота Вика в плоском пространстве и пространстве де Ситтера состоит в том, что в последнем случае меняется топология пространства:

$$
\begin{array}{ccc}
d s^{2}=d X_{d+1}^{2}-\sum_{i=1}^{d} d X_{i}^{2}, & d s^{2}=-d X_{d+1}^{2}-\sum_{i=1}^{d} d X_{i}^{2}, \\
X_{d+1}^{2}-\sum_{i=1}^{d} X_{i}^{2}=-R^{2}, & \mapsto & X_{d+1}^{2}+\sum_{i=1}^{d} X_{i}^{2}=+R^{2} .
\end{array}
$$

То же преобразование, что в (1)-(4), можно провести и в этом случае с минимальными изменениями. Производные $\partial_{\mu}$ в $(1)$ заменяются на ковариантные производные $\nabla_{\mu}$. В работе [17] было показано, что в соотношении (4) образуется дополнительный подынтегральный множитель $e^{T((d-1) / 2 r)^{2}}$. Мы можем избавиться от этого постоянного множителя, сдвинув массу:

$$
\widetilde{m}^{2}=m^{2}-\left(\frac{d-1}{2 r}\right)^{2}
$$

Мы начнем с простейшего случая двумерного пространства де Ситтера без взаимодействия. Мы можем записать ширину распада вакуума $\Gamma_{\text {Еuсl }}$ с ненулевой мнимой частью (из-за фонового гравитационного поля), следуя соотношению (4):

$$
\Gamma_{\text {Eucl }}=\int_{0}^{\infty} \frac{d T}{T} e^{-\widetilde{m}^{2} T} \int_{x_{\mu}(0)=x_{\mu}(1)} \mathcal{D} x_{\mu}(\tau) \exp \left[-\int_{0}^{1} d \tau \frac{\dot{x}^{2}}{4 T}\right] .
$$

Использование метода перевала для континуального интеграла отвечает нахождению лидирующего вклада в пределе $m r \rightarrow \infty$. Мы замечаем, что у действия есть несколько седловых точек. Они отвечают классическим траекториям, $l$ раз намотанным на экватор. На следующем шаге удобно разбить интеграл по замкнутым траекториям на интеграл с граничными условиями Дирихле и интеграл по граничным условиям. Вероятность распада в однородном пространстве пропорциональна его объему, поэтому будет удобно сначала выделить этот множитель. Интеграл

$$
\Gamma_{\text {Eucl }}=\int_{0}^{\infty} \frac{d T}{T} e^{-\widetilde{m}^{2} T} \int d^{2} \tilde{x} \int_{x_{\mu}(0)=x_{\mu}(1)} \mathcal{D} x_{\mu}(\tau) \delta^{(2)}\left(x_{\mu}(0)-\tilde{x}\right) \exp \left[-\int_{0}^{1} d \tau \frac{\dot{x}^{2}}{4 T}\right]
$$

не зависит от $\tilde{x}$, поэтому можно выбрать $\tilde{x}=0$. Для вычисления удобно ввести стандартные угловые координаты

$$
x^{\mu}=\left(\begin{array}{c}
r \phi \\
r \theta
\end{array}\right), \quad X_{0}=r \sin \theta \cos \phi, \quad X_{1}=r \sin \theta \sin \phi, \quad X_{2}=r \cos \theta .
$$


В этой системе координат классические траектории записываются как

$$
X_{(l)}^{\mu}(\tau)=\left(\begin{array}{c}
2 \pi r \tau l \\
\pi r / 2
\end{array}\right)
$$

Так как наши траектории замкнуты, мы можем разложить $x^{\mu}(\tau)$ в окрестности этих траекторий в ряд Фурье:

$$
x^{\mu}(\tau)=X_{(l)}^{\mu}+\frac{\alpha_{0}}{\sqrt{T}}+\sqrt{\frac{2}{T}} \sum_{n=1}^{\infty}\left(\alpha_{n}^{\mu} \cos (2 \pi \tau n)+\tilde{\alpha}_{n}^{\mu} \sin (2 \pi \tau n)\right) .
$$

Такая нормировка окажется удобной для будущих вычислений, найденные физические величины от нее не зависят. От дельта-функции можно избавиться при помощи стандартного приема, использовав равенство

$$
\delta^{(2)}\left(x_{\mu}\right)=\int \frac{d^{2} p_{\mu}}{(2 \pi)^{2}} e^{-i p_{\mu} x^{\mu}} .
$$

Положим

$$
S_{1 \delta}=\int_{0}^{1} d \tau \frac{\dot{x}^{2}}{4 T}+i p_{\mu} x^{\mu}(0),
$$

тогда действие (7) в приближении метода перевала можно окончательно записать каK

$$
\Gamma_{\text {Eucl }}=-V \int_{0}^{\infty} \frac{d T}{T} e^{-\widetilde{m}^{2} T} \sum_{l}\left[\operatorname{det}\left(\delta^{2} S_{1 \delta}\left(X_{(l)}\right)\right)\right]^{-1 / 2} e^{-S\left(X_{(l)}\right)},
$$

где $V$ - размер описываемой системы.

Следует сделать замечание по поводу меры интегрирования. В кривом пространстве мера

$$
\mathcal{D} x(\tau)=N \prod_{i} d x^{\mu}\left(\tau_{i}\right) \sqrt{g\left(x\left(\tau_{i}\right)\right)}
$$

включает метрический детерминант $\sqrt{g(x)}$, который равен единице на траекториях (9). Символом $\delta$ мы обозначаем вариацию по всем переменным $\alpha_{n}^{\mu}, \tilde{\alpha}_{n}^{\mu}$ и $p^{\mu}$, так как мера (12) равна произведению интегралов по коэффициентам Фурье:

$$
\mathcal{D} x(\tau)=N \prod_{n, \mu} d \alpha_{n}^{\mu} d \tilde{\alpha}_{n}^{\mu} .
$$

На интеграл в окрестности тривиальной точки перевала $X_{(0)}$ компактность и кривизна пространства не оказывают влияния, поэтому для определения нормировочного множителя $N$ можно выбрать стандартную регуляризационную схему [18]

$$
\left[\operatorname{det}\left(\delta^{2} S_{1 \delta}\left(X_{(l)}\right)\right)\right]^{-1 / 2} e^{-S\left(X_{(l)}\right)}=\operatorname{Tr} \exp \left(-\frac{p^{2}}{2} T\right)=\frac{1}{2 \pi T} .
$$

С практической точки зрения нас будут интересовать только два первых слагаемых суммы в (11), поскольку классическое действие $S\left(X_{(l)}\right)$ пропорционально $l$ и, следовательно, остальные слагаемые будут экспоненциально подавлены, а слагаемое c $l=0$ дает такой же результат, как и в плоском пространстве, и вклада в $\operatorname{Im} \Gamma$ не вносит. 
Нам потребуется отрицательное собственное значение оператора $\delta^{2} S_{1 \delta}\left(X_{(0)}\right)$ (точнее, любое нечетное количество отрицательных собственных значений). Разлагая $S_{1 \delta}$ в окрестности $X_{(0)}$ как

$$
S_{1 \delta}=0+\frac{1}{T^{2}} \sum_{n=1}^{\infty} \pi^{2} n^{2}\left(\left(\alpha_{n}^{\mu}\right)^{2}+\left(\tilde{\alpha}_{n}^{\mu}\right)^{2}\right)+i \sqrt{\frac{2}{T}} \sum_{n=1}^{\infty}\left(\alpha_{n}^{\mu} p_{\mu}\right)+i p_{\mu} \frac{\alpha_{0}^{\mu}}{\sqrt{T}}
$$

мы видим, что вариационная производная $\delta^{2} S_{1 \delta}\left(X_{(0)}\right)$ положительно определена и распадается на два слагаемых, которые отвечают двум ортогональным направлениям с $\mu=1,2$. Это означает, что детерминант распадается в произведение двух независимых частей, соответствующих этим направлениям:

$$
\operatorname{det}\left(\delta^{2} S_{1 \delta}\left(X_{(0)}\right)\right)=\operatorname{det}\left(\delta_{1} \delta_{1} S_{1 \delta}\left(X_{(0)}\right)\right) \operatorname{det}\left(\delta_{2} \delta_{2} S_{1 \delta}\left(X_{(0)}\right)\right) .
$$

Как мы видим из разложения в окрестности $X_{(1)}$,

$$
\begin{aligned}
S_{1 \delta}= & \frac{\pi^{2} r^{2}}{T}+\frac{1}{T^{2}} \sum_{n=1}^{\infty} \pi^{2} n^{2}\left(\left(\alpha_{n}^{1}\right)^{2}+\left(\tilde{\alpha}_{n}^{1}\right)^{2}+\left(\left(\alpha_{n}^{2}\right)^{2}+\left(\tilde{\alpha}_{n}^{2}\right)^{2}\right)\left(1-\frac{1}{n^{2}}\right)\right)- \\
& +\frac{\pi^{2}\left(\alpha_{0}^{2}\right)^{2}}{T^{2}}+i \sqrt{\frac{2}{T}} \sum_{n=1}^{\infty}\left(\alpha_{n}^{\mu} p_{\mu}\right)+i p_{\mu} \frac{\alpha_{0}^{\mu}}{\sqrt{T}},
\end{aligned}
$$

последнее утверждение о том, что детерминант распадается в произведение, остается в силе. Также в этом случае есть одно отрицательное собственное значение. Однако есть и нулевая мода, отсутствующая в плоском случае: квадратичная часть действия не зависит от $\tilde{\alpha}_{1}^{2}$. С отрицательной модой мы поступаем, как в работе [2]:

$$
\int_{-\infty}^{-\infty} d z e^{|a| z^{2}} \mapsto \frac{i}{2} \int_{-\infty}^{-\infty} d z e^{-|a| z^{2}}
$$

Интеграл по $\alpha_{1}^{2}$ в континуальном интеграле имеет четкую геометрическую интерпретацию. Даже зафиксировав граничные условия $x^{\mu}(0)=x^{\mu}(1)=0$, мы все равно оставляем одну степень свободы, поскольку $S O(3)$-симметрия 2-сферы сохраняет действие. Однако мы можем параметризовать эти решения точкой пересечения $\tilde{\phi}$ с трансверсальным экватором $x^{1}= \pm r \pi / 2$. Поэтому интеграл по $d \alpha_{1}^{2}$ только кажется бесконечным, на самом деле это интеграл по кольцу с учетом коэффициента пропорциональности между $\alpha_{1}$ и угловой координатой $\tilde{\phi}$,

$$
d \alpha_{1}=r \sqrt{\frac{T}{2}} d \tilde{\phi}
$$

который восстанавливается из разложения (10). Каждое классическое решение пересекает трансверсальный экватор в двух точках $\tilde{\phi}$ и $\tilde{\phi}+\pi$, но каждая траектория имеет два направления, поэтому интегрировать надо по всем значениям $\tilde{\phi}$.

Принимая во внимание все вышесказанное, мы непосредственно получаем следующую формулу:

$$
\begin{aligned}
\operatorname{Im} \Gamma_{\text {Eucl }}= & \int_{0}^{\infty} \frac{d T}{T} e^{-\widetilde{m}^{2} T-S_{1 \delta}\left(X_{(1)}\right)} \times \\
& \times\left[\operatorname{det}\left(\delta_{1} \delta_{1} S_{1 \delta}\left(X_{(1)}\right)\right) \operatorname{det}^{\prime}\left(\delta_{2} \delta_{2} S_{1 \delta}\left(X_{(1)}\right)\right)\right]^{-1 / 2} \frac{1}{\sqrt{\pi}} r \sqrt{\frac{T}{2}} d \tilde{\phi},
\end{aligned}
$$


где det' означает детерминант, для которого отброшены нулевые моды, а для отрицательных мод применено соотношение (16) (более подробно см. в приложении А). После взятия интеграла по $T$ мы получаем

$$
\begin{aligned}
\operatorname{Im} \Gamma_{\text {Eucl }} & =\int_{0}^{\infty} \frac{d T}{T} e^{-\widetilde{m}^{2} T-\pi^{2} r^{2} / T} \frac{V r \sqrt{\pi}}{2 T^{3 / 2}}= \\
& =\frac{V}{4 \pi^{2} r^{2}}(1+2 \pi \widetilde{m} r) e^{-2 \pi \widetilde{m} r} \approx \frac{4 \widetilde{m} V}{\pi r} e^{-2 \pi \widetilde{m} r}
\end{aligned}
$$

Показатель экспоненты в правой части этой формулы совпадает с выражением, приведенным в работе [12].

\section{3. МНИМАЯ ЧАСТЬ ДВУХТОЧЕЧНОЙ СКАЛЯРНОЙ ФУНКЦИИ В ПРОСТРАНСТВЕ ДЕ СИТТЕРА}

Цель этого раздела - найти лидирующий мнимый вклад в однопетлевую двухточечную амплитуду в теории массивного скалярного поля с потенциалом

$$
U(\phi)=\frac{\lambda}{3 !} \phi^{3}
$$

Двухточечная амплитуда определяет скорость распада скалярных частиц вследствие внутрипетлевого взаимодействия с фоновым гравитационным полем.

Мы начнем с двумерного случая, но в разделе 4 обсудим случай произвольной размерности. Двухточечная амплитуда может быть определена [19], [20] как вариация статистической суммы $Z[\phi]=e^{\Gamma[\phi]}$ :

$$
\Pi(y, z)=\frac{1}{Z} \frac{\delta}{\delta \phi(y)} \frac{\delta}{\delta \phi(z)} Z=\frac{\delta}{\delta \phi(y)} \frac{\delta \Gamma}{\delta \phi(z)}+\frac{\delta \Gamma}{\delta \phi(y)} \frac{\delta \Gamma}{\delta \phi(z)} .
$$

Поскольку второе слагаемое не зависит от расстояния между $y$ и $z$, мы можем его сразу отбросить. Используя равенство

$$
\frac{\delta}{\delta \phi(y)} \int_{0}^{1} d \tau \phi(x(\tau))=\int_{0}^{1} d \tau \delta^{2}(x-y)
$$

мы можем переписать формулу (19) как

$$
\Pi(y, z)=\lambda^{2} \int_{0}^{\infty} \frac{d T}{T} e^{-\widetilde{m}^{2} T} \int \frac{d^{2} p d^{2} q}{(2 \pi)^{4}} T^{2} \int_{0}^{1} d \tau^{\prime} \int_{0}^{1} d \tau^{\prime \prime} \int \mathcal{D} x(\tau) e^{S_{2 \delta}-i p y-i q z}
$$

где в величину

$$
S_{2 \delta}=\int_{0}^{1} d \tau \frac{\dot{x}^{2}}{4 T}+i p_{\mu} x^{\mu}\left(\tau^{\prime}\right)+i q_{\mu} x^{\mu}\left(\tau^{\prime \prime}\right)
$$

мы собрали все слагаемые с квадратичными вкладами. Данное выражение также содержит линейный вклад $i p_{\mu} X_{(1)}^{\mu}\left(\tau^{\prime}\right)+i q_{\mu} X_{(1)}^{\mu}\left(\tau^{\prime \prime}\right)$. 
Континуальный интеграл по-прежнему является гауссовым, хотя теперь в нем присутствует линейный сдвиг:

$$
\int d \vec{\alpha} e^{-\vec{\alpha}^{\mathrm{T}} \delta^{2} S \vec{\alpha}-i \vec{J} \vec{\alpha}}=\left[\operatorname{det}\left(\delta^{2} S\right)\right]^{-1 / 2} e^{-\vec{J}\left(\delta^{2} S\right)^{-1} \vec{J}^{\mathrm{T}}} .
$$

Данная запись несколько схематична, но она передает общую идею - вектор коллективных координат $\vec{\alpha}$ содержит все переменные, по которым мы интегрируем в бесконечных пределах. Для упрощения вычисления удобно выбрать точки $y$ и $z$ как

$$
y^{\mu}=\left(\begin{array}{c}
r \phi_{1} \\
\pi r / 2
\end{array}\right), \quad z^{\mu}=\left(\begin{array}{c}
r \phi_{2} \\
\pi r / 2
\end{array}\right),
$$

поскольку мы знаем, что коррелятор зависит только от расстояния $r\left(\phi_{2}-\phi_{1}\right)$ между $у$ и $z$. Детерминант для континуального интеграла

$$
\operatorname{det}^{\prime}\left(\delta^{2} S_{2 \delta}\left(X_{(1)}\right)\right)=4 T^{4} \sin ^{2}(2 \pi \tau) \tau(1-\tau)
$$

не зависит от $\tau^{\prime}$ и $\tau^{\prime \prime}$, а зависит только от разности $\tau=\tau^{\prime}-\tau^{\prime \prime}$. Он равен нулю для $\tau=0,1 / 2,1$ (более подробно см. в приложении А). Также стоит отметить что для $\tau=1 / 2$ можно регуляризовать этот детерминант так же, как мы делали это в разделе 2:

$$
\left[\operatorname{det}^{\prime}\left(\delta^{2} S_{2 \delta}\left(X_{(1)}\right)\right)\right]^{-1 / 2} \int d \alpha=\left(\frac{2}{\pi^{2}} T^{6} \tau(1-\tau)\right)^{-1 / 2} \int d \tilde{\phi} r \sqrt{\frac{T}{2}} .
$$

Здесь нет отрицательных собственных значений, поэтому нет вклада в мнимую часть коррелятора. Геометрическая причина этого проста: значение $\tau=1 / 2$ отвечает траекториям, проходящим через противоположные точки на сфере. Хотя существует множество этих траекторий с одинаковым действием, такую траекторию нельзя деформировать в более короткую.

Выполнив в $(20)$ интегрирование по $\mathcal{D} x^{1}(\tau)$, мы получаем квадратичный вклад в эффективное действие:

$$
-\frac{1}{4} \vec{J}_{1}\left(\delta_{1}^{2} S_{2 \delta}\left(X_{(1)}\right)\right)^{-1} \vec{J}_{1}^{\mathrm{T}}=\frac{-r^{2}\left(\left(\phi_{1}-2 \pi \tau^{\prime}\right)^{2}+\left(\phi_{2}-2 \pi \tau^{\prime \prime}\right)^{2}\right)}{4 T \tau(1-\tau)} .
$$

После интегрирования по $d \tau^{\prime}$ и $d \tau^{\prime \prime}$ при помощи метода перевала, вследствие того что минимум экспоненциальной части находится в точке с координатами $\tau^{\prime}=\phi_{1} / 2 \pi$, $\tau^{\prime \prime}=\phi_{2} / 2 \pi$, этот вклад в действие пропадает. В результате получаем множитель $\pi T \tau(1-\tau) / 4$. Для $\tau \neq 1 / 2$ и $\tau=1 / 2$ соответственно имеем

$$
\begin{aligned}
\int_{0}^{\infty} d T e^{-m^{2} T-\pi^{2} r^{2} / T} & \approx \frac{\pi r}{m} e^{-2 \pi m r} \sqrt{\frac{1}{m r}} \\
\int_{0}^{\infty} \frac{d T}{\sqrt{T}} e^{-m^{2} T-\pi^{2} r^{2} / T} & =e^{-2 \pi m r} \frac{\sqrt{\pi}}{m} .
\end{aligned}
$$

Это дает нам выражение для лидирующего вклада в двухточечный коррелятор:

$$
\operatorname{Im} \Pi(y, z)=\frac{\lambda^{2}}{16 \pi^{2}(\widetilde{m} r)^{3 / 2}} \frac{\sqrt{|y-z|(2 \pi r-|y-z|)}}{r|\sin (|y-z| / r)|} e^{-2 \pi \widetilde{m} r} .
$$


То, что это выражение имеет полюс в точке $|y-z|=\pi r$, противоречит прямому вычислению для $|y-z|=\pi r$ с детерминантом (23), которое дает нам поправку к действительной части

$$
\Delta \operatorname{Re} \Pi\left(y, y+\left(\begin{array}{c}
\pi r \\
0
\end{array}\right)\right)=\frac{\lambda^{2}}{16 \widetilde{m} r} e^{-2 \pi \widetilde{m} r}
$$

В окрестности точки $|y-z|=\pi r$ одно из собственных значений оператора $\delta^{2} S_{2 \delta}\left(X_{(1)}\right)$ стремится к нулю, поэтому становится невозможным применять гауссово приближение. Но для области $\pi-|y-z| / r \ll 1$ мы можем предложить интерполяцию, совершив замену

$$
\left|\sin \left(\frac{|y-z|}{r}\right)\right|^{-1} \mapsto \operatorname{Re}\left(\left|\sin \frac{|y-z|}{r}\right|+i \frac{1}{\pi \sqrt{\tilde{m} r}}\right)^{-1} .
$$

\section{4. СЛУЧАЙ ПРОИЗВОЛЬНОЙ РАЗМЕРНОСТИ}

Цель этого раздела состоит в том, чтобы применить рассмотренные выше методы к $d$-мерному пространству де Ситтера (см. соотношения (6)). Введя координаты, аналогичные (8),

$x^{\mu}=\left(\begin{array}{c}r \phi \\ r \vec{\theta}\end{array}\right), \quad X_{0}=r(\vec{\theta}) \cos \phi, \quad X_{1}=r A(\vec{\theta}) \sin \phi, \quad X_{i}=r \cos \theta_{i}, \quad i=2, \ldots, d$,

можно получить классические траектории

$$
X_{(l)}^{\mu}(\tau)=\left(\begin{array}{c}
2 \pi r \tau l \\
\pi r \overrightarrow{1} / 2
\end{array}\right)
$$

которые обобщают траектории (9). Классическое действие на этих траекториях не зависит от $d$. Разложим действие в окрестности $X_{(1)}^{\mu}(\tau)$,

$$
\begin{aligned}
& S_{1 \delta}=\frac{\pi^{2} r^{2}}{T}+\frac{1}{T^{2}} \sum_{n=1}^{\infty} \pi^{2} n^{2}\left(\left(\alpha_{n}^{1}\right)^{2}+\left(\tilde{\alpha}_{n}^{1}\right)^{2}\right)+ \\
& +\frac{1}{T^{2}} \sum_{\substack{n=1, \nu>2}}^{\infty} \pi^{2} n^{2}\left(\left(\left(\alpha_{n}^{\nu}\right)^{2}+\left(\tilde{\alpha}_{n}^{\nu}\right)^{2}\right)\left(1-\frac{1}{n^{2}}\right)\right)-\frac{\pi^{2}\left(\alpha_{0}^{2}\right)^{2}}{T^{2}}+i \sqrt{\frac{2}{T}} \sum_{n=1}^{\infty}\left(\alpha_{n}^{\mu} p_{\mu}\right)+i p_{\mu} \frac{\alpha_{0}^{\mu}}{\sqrt{T}},
\end{aligned}
$$

получим сумму $d$ независимых частей, $d-1$ из которых равны друг другу. Это означает, что если мы возьмем континуальный интеграл (7) с помощью метода перевала (11), то $\operatorname{det}\left(\delta^{2} S_{1 \delta}\left(X_{(l)}\right)\right)$ распадется в произведение:

$$
\operatorname{det}^{\prime}\left(\delta^{2} S_{1 \delta}\left(X_{(l)}\right)\right)=\operatorname{det}\left(\delta_{1}^{2} S_{1 \delta}\left(X_{(l)}\right)\right)\left[\operatorname{det}\left(\delta_{2}^{2} S_{1 \delta}\left(X_{(l)}\right)\right)\right]^{d-1} .
$$

Применим процедуру (16), тогда каждый детерминант $\operatorname{det}^{\prime}\left(\delta_{2}^{2} S_{1 \delta}\left(X_{(l)}\right)\right)$ даст множитель $i$, поэтому для нечетных $d$ все вклады действительные, и рождения частиц не происходит. В терминах вакуума Банч-Дэвиса это впервые было замечено в работе [15]. Поэтому все дальнейшие формулы мы будем писать для четных значений $d$. 


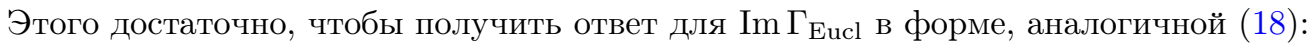

$$
\begin{aligned}
\operatorname{Im} \Gamma_{\text {Eucl }}= & V \int_{0}^{\infty} \frac{d T}{T} e^{-\widetilde{m}^{2} T-S_{1 \delta}\left(X_{(1)}\right)}\left[\operatorname{det}\left(\delta_{1}^{2} S_{1 \delta}\left(X_{(1)}\right)\right) \operatorname{det}\left(\delta_{2}^{2} S_{1 \delta}\left(X_{(1)}\right)\right)\right]^{-1 / 2} \times \\
& \times \int d \Omega_{d-1}\left(\frac{T r^{2}}{2 \pi}\right)^{(d-1) / 2} .
\end{aligned}
$$

Здесь $d \Omega_{d-1}$ - мера на $(d-1)$-мерной сфере и $V$ - объем пространства. Причина замены $(d-1)$-мерного интеграла по нулевым модам та же, что и в разделе 2 . Множество классических решений может быть параметризовано точкой пересечения со сферой $x^{1}= \pm r \pi / 2$. Каждая траектория проходит через эту точку дважды, но у каждой траектории есть два направления. Имеем

$$
\begin{aligned}
\Gamma_{d} & =2^{1-3 d / 2} \pi^{d / 2-1 / 2} r^{d-1} \frac{d}{(d / 2) !} \int_{0}^{\infty} \frac{\sqrt{T} d T}{T^{d+1}} e^{-\widetilde{m}^{2} T-\pi^{2} r^{2} / T} \approx \\
& \approx \frac{d}{(d / 2) !} \frac{2^{1-d}}{(2 \pi)^{d / 2}} \frac{\widetilde{m}^{d-1}}{r} e^{-2 \pi \widetilde{m} r} .
\end{aligned}
$$

Размерная часть преэкспоненциального фактора $\widetilde{m}^{d-1} / r$ отличается от ожидаемой $(\widetilde{m} / r)^{d / 2}$, которая получается по аналогии с (5). Это можно объяснить следующим образом. Физическая размерность зафиксирована, если фиксирован объем. В случае присутствия электрического поля масса в преэкспоненциальный фактор не входит. Заряд и поле всегда входят в виде произведения, поэтому для (5) ответ зафиксирован. В нашем случае эффективный заряд $m$ и эффективное поле $r$ могут входить в разных комбинациях. Мы исследуем предел $m r \rightarrow \infty$ и ищем лидирующий вклад. Вклад, пропорциональный $(\widetilde{m} / r)^{d / 2}$, присутствует, но подавлен.

Лидирующий вклад в мнимую часть двухточечного коррелятора можно вычислить аналогично. Выбрав аргументы схожим образом с (22), мы можем использовать формулу (24), если заменить двумерный детерминант $d$-мерным. Снова вклад данного слагаемого мнимый только для четного $d$ :

$$
\begin{aligned}
\operatorname{det}^{\prime}\left(\delta^{2} S_{2 \delta}\left(X_{(1)}\right)\right) & =\operatorname{det}^{\prime}\left(\delta_{1} \delta_{1} S_{2 \delta}\left(X_{(1)}\right)\right)\left[\operatorname{det}^{\prime}\left(\delta_{2} \delta_{2} S_{2 \delta}\left(X_{(1)}\right)\right)\right]^{d-1}= \\
& =\left(\frac{2 T^{2}}{\pi}\right)^{d} \pi^{2} \tau(1-\tau)\left(\sin ^{2}(2 \pi \tau)\right)^{d-1} .
\end{aligned}
$$

Интегралы по $d T$ теперь имеют вид

$$
\begin{aligned}
\int_{0}^{\infty} \frac{d T}{T^{d-2}} e^{-m^{2} T-\pi^{2} r^{2} / T} & =2 \frac{m^{d-3}}{(\pi r)^{d-3}} K_{d-3}(2 \pi m r) \approx \\
& \approx \frac{m^{d-3}}{(\pi r)^{d-3}} e^{-2 m \pi r} \sqrt{\frac{1}{m r}}
\end{aligned}
$$

В результате получаем окончательный ответ:

$$
\operatorname{Im} \Pi(y, z)=\lambda^{2} \frac{\widetilde{m}^{d-7 / 2} \pi^{2-2 d}}{r^{d+1 / 2} 2^{5 d / 2-1}} \frac{\sqrt{|y-z|(2 \pi r-|y-z|)}}{|\sin (|y-z| / r)|^{d-1}} e^{-2 \pi \widetilde{m} r}
$$




\section{5. ВЫВОДЫ}

Исследованы процессы спонтанного и индуцированного рождения частиц во внешнем гравитационном поле. Мы работали со скалярной теорией поля в пространстве де Ситтера. Для вероятности спонтанного рождения пар найден метод получения преэкспоненциального фактора. Среди преимуществ этого метода - простота обобщения на случай произвольной размерности. Этот метод также дает простое геометрическое объяснение того факта, что в случае нечетного количества измерений в пределе слабого поля эффект пропадает.

Предложено обобщение данного метода для вычисления лидирующего вклада в двухточечный коррелятор, который описывает распад частиц. В отличие от схожего процесса во внешнем электрическом поле, в пространстве де Ситтера экспоненциальное подавление остается без изменения.

\section{Вычисление детерминантов}

ПРИЛОЖКНИЕ А

В этом разделе мы приведем подробное вычисление бесконечных детерминантов, появляющихся выше. Как мы уже отмечали, мы выбираем регуляризационную схему (13). Это позволит нам формально заменять бесконечное расходящееся произведение некоторым конечным выражением. Чтобы так поступить, необходимо сначала найти этот ответ для какого-то одного случая, чтобы принять его за определение. Как видно из разложений (14) и (15),

$$
\operatorname{det}\left(\delta_{1} \delta_{1} S_{1 \delta}\left(X_{(0)}\right)\right)=\operatorname{det}\left(\delta_{2} \delta_{2} S_{1 \delta}\left(X_{(0)}\right)\right)=\operatorname{det}\left(\delta_{1} \delta_{1} S_{1 \delta}\left(X_{(1)}\right)\right)=2 \pi T .
$$

Продольный детерминант не изменяется при смене седловой точки. Чтобы вычислить $\operatorname{det}\left(\delta_{1} \delta_{1} S_{1 \delta}\left(X_{(1)}\right)\right)$, нам будет удобно выбрать следующий порядок переменных: $p^{1}, \alpha_{0}^{1}, \alpha_{1}^{1}, \tilde{\alpha}_{1}^{1}, \ldots, \alpha_{n}^{1}, \tilde{\alpha}_{n}^{1}, \ldots$. При таком выборе матрицу $\delta_{1} \delta_{1} S_{1 \delta}\left(X_{(1)}\right)$ можно записать как

$$
\delta_{1} \delta_{1} S_{1 \delta}\left(X_{(1)}\right)=\left[\begin{array}{ccccccc}
0 & \frac{i}{2 \sqrt{T}} & \frac{i}{\sqrt{2 T}} & 0 & \frac{i}{\sqrt{2 T}} & 0 & \ldots \\
\frac{i}{2 \sqrt{T}} & 0 & 0 & 0 & 0 & 0 & \ldots \\
\frac{i}{\sqrt{2 T}} & 0 & \frac{\pi^{2}}{T^{2}} & 0 & 0 & 0 & \ldots \\
0 & 0 & 0 & \frac{\pi^{2}}{T^{2}} & 0 & 0 & \ldots \\
\frac{i}{\sqrt{2 T}} & 0 & 0 & 0 & \frac{4 \pi^{2}}{T^{2}} & 0 & \ldots \\
\vdots & \vdots & \vdots & \vdots & \vdots & \vdots & \ddots
\end{array}\right] .
$$

Структура этой матрицы достаточно удобна, хотя она и не является диагональной. Матрицу можно разбить на блоки:

$$
\delta_{1} \delta_{1} S_{1 \delta}\left(X_{(1)}\right)=\left[\begin{array}{cc}
\mathbf{A}_{k \times k} & \mathbf{B}_{k \times \infty} \\
\mathbf{B}_{\infty \times k}^{\mathrm{T}} & \mathbf{D}_{\infty \times \infty}
\end{array}\right],
$$

где индексы блока обозначают его размер. Если мы выберем $k=2$, то блок $\mathbf{D}$ станет диагональным и обратимым. Эти свойства $\mathbf{D}$ останутся верными для матриц 
с другими $k$. Так как матрица $\mathbf{D}$ диагональна и обратима, мы можем использовать метод Гаусса и сделать матрицу В нулевой без изменения детерминанта. Недостаток этого подхода в том, что матрица А заменяется матрицей $\tilde{\mathbf{A}}$. Тогда детерминант факторизуется как

$$
\left|\begin{array}{cc}
\tilde{\mathbf{A}}_{k \times k} & \mathbf{0}_{k \times \infty} \\
\mathbf{C}_{\infty \times k} & \mathbf{D}_{\infty \times \infty}
\end{array}\right|=\operatorname{det} \tilde{\mathbf{A}} \operatorname{det} \mathbf{D}
$$

Впрочем, для $\delta_{1} \delta_{1} S_{1 \delta}\left(X_{(1)}\right)$ матрицы $\tilde{\mathbf{A}}$ и $\mathbf{A}$ различаются только в левом верхнем элементе, изменение которого не оказывает влияния на детерминант. Поэтому выражение

$$
\operatorname{det}\left(\delta_{1} \delta_{1} S_{1 \delta}\left(X_{(1)}\right)\right)=N \frac{1}{4 T}\left(\prod_{n=1}^{\infty} \frac{\pi^{2} n^{2}}{T^{2}}\right)^{2}=2 \pi T
$$

дает нам определение нашей регуляризации.

В случае

$$
\delta_{2} \delta_{2} S_{1 \delta}\left(X_{(1)}\right)=\left[\begin{array}{ccccccc}
0 & \frac{i}{2 \sqrt{T}} & \frac{i}{\sqrt{2 T}} & 0 & \frac{i}{\sqrt{2 T}} & 0 & \ldots \\
\frac{i}{2 \sqrt{T}} & -\frac{\pi^{2}}{T^{2}} & 0 & 0 & 0 & 0 & \ldots \\
\frac{i}{\sqrt{2 T}} & 0 & 0 & 0 & 0 & 0 & \ldots \\
0 & 0 & 0 & 0 & 0 & 0 & \ldots \\
\frac{i}{\sqrt{2 T}} & 0 & 0 & 0 & \frac{3 \pi^{2}}{T^{2}} & 0 & \ldots \\
0 & 0 & 0 & 0 & 0 & \frac{3 \pi^{2}}{T^{2}} & \ldots \\
\vdots & \vdots & \vdots & \vdots & \vdots & \vdots & \ddots
\end{array}\right]
$$

все несколько сложнее. Минимальная матрица А из (А.1) достигается при $k=4$. $\mathrm{Y}$ нее есть одно нулевое собственное значение, отвечающее интегрированию по $\tilde{\alpha}_{1}^{2}$. С этим интегрированием следует поступить, как в (17), но это означает, что по сравнению с (А.2) у нас на один гауссов интеграл меньше. В результате появляется дополнительный множитель $1 / \sqrt{\pi}$. Элементы $\mathbf{A}_{1,1}$ и $\tilde{\mathbf{A}}_{1,1}$ также различны, но на произведение ненулевых собственных значений $-\pi^{2} / 2 T^{3}$ (детерминант левого верхнего минора размера $3 \times 3$ ) они не влияют. Учитывая процедуру (16) для отрицательных и нулевых собственных значений, мы получаем

$$
\operatorname{det}^{\prime}\left(\delta_{2} \delta_{2} S_{1 \delta}\left(X_{(1)}\right)\right)=4\left(\frac{2 \pi^{2}}{4 T^{3}}\right)\left(\prod_{n=2}^{\infty} \frac{\pi^{2}\left(n^{2}-1\right)}{T^{2}}\right)^{2}=\frac{4 T^{3}}{\pi} .
$$

Для $S_{2 \delta}$ детерминанты матриц $\mathbf{A}$ и $\tilde{\mathbf{A}}$ оказываются различными. Из-за второй дельта-функции возникает новая переменная интегрирования $q_{\mu}$, поэтому матрица $\mathbf{A}$ имеет размер $3 \times 3$ для $\delta_{1} \delta_{1} S_{2 \delta}\left(X_{(1)}\right)$ и размер $5 \times 5$ для $\delta_{1} \delta_{1} S_{2 \delta}\left(X_{(1)}\right)$. В ре- 
зультате гауссова исключения мы получаем нулевой блок В для матрицы

$$
\delta_{1} \delta_{1} S_{2 \delta}\left(X_{(1)}\right)=\left[\begin{array}{cccccc}
0 & 0 & \frac{i}{2 \sqrt{T}} & \frac{i \cos \left(2 \pi \tau^{\prime}\right)}{\sqrt{2 T}} & \frac{i \sin \left(2 \pi \tau^{\prime}\right)}{\sqrt{2 T}} & \ldots \\
0 & 0 & \frac{i}{2 \sqrt{T}} & \frac{i \cos \left(2 \pi \tau^{\prime \prime}\right)}{\sqrt{2 T}} & \frac{i \sin \left(2 \pi \tau^{\prime \prime}\right)}{\sqrt{2 T}} & \ldots \\
\frac{i}{2 \sqrt{T}} & \frac{i}{2 \sqrt{T}} & 0 & 0 & 0 & \ldots \\
\frac{i \cos \left(2 \pi \tau^{\prime}\right)}{\sqrt{2 T}} & \frac{i \cos \left(2 \pi \tau^{\prime \prime}\right)}{\sqrt{2 T}} & 0 & \frac{\pi^{2}}{T^{2}} & 0 & \ldots \\
\frac{i \sin \left(2 \pi \tau^{\prime}\right)}{\sqrt{2 T}} & \frac{i \sin \left(2 \pi \tau^{\prime \prime}\right)}{\sqrt{2 T}} & 0 & 0 & \frac{\pi^{2}}{T^{2}} & \ldots \\
\vdots & \vdots & \vdots & \vdots & \vdots & \ddots
\end{array}\right],
$$

но при этом изменяется левый верхний $(2 \times 2)$-блок матрицы $\mathbf{A}$, который превращается в

$$
\frac{T}{2}\left[\begin{array}{ll}
F(0) & F(\tau) \\
F(\tau) & F(0)
\end{array}\right]
$$

где $\tau=\tau^{\prime}-\tau^{\prime \prime}$ и

$$
F(\tau)=\sum_{n=1}^{\infty} \frac{\cos (2 \pi n \tau)}{\pi^{2} n^{2}}=\frac{1}{6}-\tau+\tau^{2}
$$

Детерминант

$$
\operatorname{det}\left(\delta_{1} \delta_{1} S_{2 \delta}\left(X_{(1)}\right)\right)=\frac{1-6 F(\tau)}{24}\left(\prod_{n=1}^{\infty} \frac{\pi^{2} n^{2}}{T^{2}}\right)^{2}=\tau(1-\tau) 2 \pi T^{2}
$$

пропорционален $T^{2}$, и это согласуется с размерностным анализом. Далее, матрица $\delta_{2} \delta_{2} S_{2 \delta}\left(X_{(1)}\right)$ равна

$$
\left[\begin{array}{cccccccc}
0 & 0 & \frac{i}{2 \sqrt{T}} & \frac{i \cos \left(2 \pi \tau^{\prime}\right)}{\sqrt{2 T}} & \frac{i \sin \left(2 \pi \tau^{\prime}\right)}{\sqrt{2 T}} & \frac{i \cos \left(4 \pi \tau^{\prime}\right)}{\sqrt{2 T}} & \frac{i \sin \left(4 \pi \tau^{\prime}\right)}{\sqrt{2 T}} & \ldots \\
0 & 0 & \frac{i}{2 \sqrt{T}} & \frac{i \cos \left(2 \pi \tau^{\prime \prime}\right)}{\sqrt{2 T}} & \frac{i \sin \left(2 \pi \tau^{\prime \prime}\right)}{\sqrt{2 T}} & \frac{i \cos \left(4 \pi \tau^{\prime \prime}\right)}{\sqrt{2 T}} & \frac{i \sin \left(4 \pi \tau^{\prime \prime}\right)}{\sqrt{2 T}} & \ldots \\
\frac{i}{2 \sqrt{T}} & \frac{i}{2 \sqrt{T}} & -\frac{\pi^{2}}{T^{2}} & 0 & 0 & \ldots & & \\
\frac{i \cos \left(2 \pi \tau^{\prime}\right)}{\sqrt{2 T}} & \frac{i \cos \left(2 \pi \tau^{\prime \prime}\right)}{\sqrt{2 T}} & 0 & 0 & 0 & 0 & 0 & \ldots \\
\frac{i \sin \left(2 \pi \tau^{\prime}\right)}{\sqrt{2 T}} & \frac{i \sin \left(2 \pi \tau^{\prime \prime}\right)}{\sqrt{2 T}} & 0 & 0 & 0 & 0 & 0 & \ldots \\
\frac{i \cos \left(4 \pi \tau^{\prime}\right)}{\sqrt{2 T}} & \frac{i \cos \left(4 \pi \tau^{\prime \prime}\right)}{\sqrt{2 T}} & 0 & 0 & 0 & \frac{3 \pi^{2}}{T^{2}} & 0 & \ldots \\
\frac{i \sin \left(4 \pi \tau^{\prime}\right)}{\sqrt{2 T}} & \frac{i \sin \left(4 \pi \tau^{\prime \prime}\right)}{\sqrt{2 T}} & 0 & 0 & 0 & 0 & \frac{3 \pi^{2}}{T^{2}} & \ldots \\
\vdots & \vdots & \vdots & \vdots & \vdots & \vdots & \vdots & \ddots
\end{array}\right]
$$

и изменение ее левого верхнего $(2 \times 2)$-блока не меняет детерминанта матрицы $\tilde{\mathbf{A}}$, поэтому

$$
\operatorname{det}^{\prime}\left(\delta_{2} \delta_{2} S_{2 \delta}\left(X_{(1)}\right)\right)=4 \frac{\pi^{2} \sin ^{2}(2 \pi \tau)}{4 T^{4}}\left(\prod_{n=2}^{\infty} \frac{\pi^{2}\left(n^{2}-1\right)}{T^{2}}\right)^{2}=\frac{2 T^{2} \sin ^{2}(2 \pi \tau)}{\pi} .
$$

Множитель 4 возникает вследствие процедуры (16). 


\section{ПРИЛОЖЕНИЕ Б}

\section{Свертка векторов с обратными матрицами}

Для вычисления двухточечного коррелятора в разделе 3 нам требуется выражение для сверток в (21). Как было отмечено в приложении А, из-за такого порядка переменных интегрирования матрицы имеют удобную структуру. Для них мы можем использовать формулу блочного обращения:

$$
\begin{aligned}
\mathbf{\Lambda}^{-1} & =\left[\begin{array}{cc}
\mathbf{A} & \mathbf{B} \\
\mathbf{B}^{\mathrm{T}} & \mathbf{D}
\end{array}\right]^{-1}= \\
& =\left[\begin{array}{cc}
\left(\mathbf{A}-\mathbf{B D}^{-1} \mathbf{B}^{\mathrm{T}}\right)^{-1} & -\left(\mathbf{A}-\mathbf{B D}^{-1} \mathbf{B}^{\mathrm{T}}\right)^{-1} \mathbf{B D}^{-1} \\
-\mathbf{D}^{-1} \mathbf{B}^{\mathrm{T}}\left(\mathbf{A}-\mathbf{B D}^{-1} \mathbf{B}^{\mathrm{T}}\right)^{-1} & \mathbf{D}^{-1}+\mathbf{D}^{-1} \mathbf{B}^{\mathrm{T}}\left(\mathbf{A}-\mathbf{B D}^{-1} \mathbf{C}\right)^{-1} \mathbf{B D}^{-1}
\end{array}\right]
\end{aligned}
$$

если матрицы $\mathbf{D}$ и $\left(\mathbf{A}-\mathbf{B D}^{-1} \mathbf{B}^{\mathrm{T}}\right)$ обратимы. Для нашего выбора переменных нам нужно найти только свертку $\vec{J}\left(\delta_{1} \delta_{1} S_{2 \delta}\left(X_{(1)}\right)\right)^{-1} \vec{J}^{\mathrm{T}}$, где

$$
\vec{J}=\left(\phi_{1}-2 \pi \tau^{\prime}, \phi_{2}-2 \pi \tau^{\prime \prime}, 0, \ldots\right) .
$$

Единственный нужный нам блок матрицы $\delta_{1} \delta_{1} S_{2 \delta}\left(X_{(1)}\right)$ есть

$$
\left(\mathbf{A}-\mathbf{B D}^{-1} \mathbf{B}^{\mathrm{T}}\right)^{-1}=\left[\begin{array}{ccc}
\frac{1}{T \tau(1-\tau)} & \frac{-1}{T \tau(1-\tau)} & -i \sqrt{T} \\
\frac{-1}{T \tau(1-\tau)} & \frac{1}{T \tau(1-\tau)} & -i \sqrt{T} \\
-i \sqrt{T} & -i \sqrt{T} & T^{2}\left(\tau^{2}-\tau+1 / 3\right)
\end{array}\right]
$$

Конечное выражение имеет вид

$$
\vec{J}_{1}\left(\delta_{1}^{2} S_{2 \delta}\left(X_{(1)}\right)\right)^{-1} \vec{J}_{1}^{\mathrm{T}}=\frac{r^{2}\left(\left(\phi_{1}-2 \pi \tau^{\prime}\right)^{2}+\left(\phi_{2}-2 \pi \tau^{\prime \prime}\right)^{2}\right)}{T \tau(1-\tau)} .
$$

Благодарности. Автор благодарит А. Горского, Э. Ахмедова и П. Сатунина за ценные обсуждения. Работа выполнена при частичной финансовой поддержке РФФИ (гранты № 12-02-00284, 14-02-31768 и 12-02-91052-НЦНИ_а).

\section{Список литературы}

[1] S. R. Coleman, Phys. Rev. D, 15:4 (1977), 2929-2936; Erratum 16:4 (1977), 1248.

[2] C. G. Callan, Jr., S. R. Coleman, Phys. Rev. D, 16:6 (1977), 1762-1768.

[3] М. Б. Волошин, И. Ю. Кобзарев, Л. Б. Окунь, ЯФ, 20:6 (1974), 1229-1234.

[4] М. Б. Волошин, К. Г. Селиванов, Писъма в ЖЖЭТФ, 42:8 (1985), 342-345.

[5] J. Schwinger, Phys. Rev. (2), 82:5 (1951), 664-679.

[6] I. K. Affleck, O. Alvarez, N. S. Manton, Nucl. Phys. B, 197:3 (1982), 509-519.

[7] G. V. Dunne, Q.-H. Wang, H. Gies, C. Schubert, Phys. Rev. D (3), 73:6 (2006), 065028, 13 pp., arXiv: hep-th/0602176.

[8] K. Bulycheva, S. Guts, Phys. Rev. D, 88:5 (2013), 053001, 6 pp., arXiv: 1107.1428.

[9] A. Monin, M. B. Voloshin, Phys. Rev. D, 81:2 (2010), 025001, 6 pp., arXiv: 0910.4762.

[10] A. Gorsky, M. B. Voloshin, Phys. Rev. D, 73:2 (2006), 025015, 9 pp., arXiv: hep-th/0511095.

[11] A. D. Mironov, A. Yu. Morozov, T. N. Tomaras, Писъма в ЖКЭТФ, 94:11 (2011), 872-876, arXiv: 1108.2821. 
[12] D. Krotov, A. M. Polyakov, Nucl. Phys. B, 849:2 (2011), 410-432, arXiv: 1012.2107.

[13] E. T. Akhmedov, F. K. Popov, V. M. Slepukhin, Phys. Rev. D, 88:2 (2013), 024021, 10 pp., arXiv: 1303.1068 .

[14] C. Schubert, Phys. Rep., 355:2-3 (2001), 73-234, arXiv: hep-th/0101036.

[15] R. Bousso, A. Maloney, A. Strominger, Phys. Rev. D (3), 65:10 (2002), 104039, 24 pp., arXiv: hep-th/0112218.

[16] F. Bastianelli, A. Zirotti, Nucl. Phys. B, 642:1-2 (2002), 372-388, arXiv: hep-th/0205182.

[17] C. Grosche, F. Steiner, Ann. Phys., 182:1 (1988), 120-156.

[18] А. И. Вайнтштейн, В. И. Захаров, В. А. Новиков, М. А. Шифман, УФН, 136:4 (1982), $553-591$.

[19] A. Monin, M. B. Voloshin, Semiclassical calculation of an induced decay of false vacuum, arXiv: 1004.2015 .

[20] P. Satunin, Phys. Rev. D, 87:10 (2013), 105015, 7 pp., arXiv: 1301.5707.

Поступила в редакцию 16.12.2013 